\title{
TCP and iso-stationary transformations
}

\author{
J.S.H. van Leeuwaarden • A.H. Löpker · T.J. Ott
}

Received: 12 December 2008 / Revised: 19 October 2009 / Published online: 14 November 2009

(C) The Author(s) 2009. This article is published with open access at Springerlink.com

\begin{abstract}
We consider piecewise-deterministic Markov processes that occur as scaling limits of discrete-time Markov chains that describe the Transmission Control Protocol (TCP). The class of processes allows for general increase and decrease profiles. Our key observation is that stationary results for the general class follow directly from the stationary results for the idealized TCP process. The latter is a Markov process that increases linearly and experiences downward jumps at times governed by a Poisson process. To establish this connection, we apply space-time transformations that preserve the properties of the class of Markov processes.
\end{abstract}

Keywords Piecewise-deterministic Markov process - Time transformation · Space-time change $\cdot$ Stationary distribution $\cdot$ TCP

Mathematics Subject Classification (2000) $60 \mathrm{~J} 25 \cdot 60 \mathrm{~K} 10 \cdot 60 \mathrm{~K} 30 \cdot 90 \mathrm{~B} 15$

\section{Introduction}

This paper deals with a class $\mathcal{M}\left(r, \alpha ; \lambda, \beta ; F_{Q}\right)$ of piecewise-deterministic Markov processes (PDMPs) related to the Transmission Control Protocol (TCP) for data

J.S.H. van Leeuwaarden $(\bowtie) \cdot$ A.H. Löpker

Eindhoven University of Technology and EURANDOM, P.O. Box 513, 5600 MB Eindhoven, The Netherlands

e-mail: j.s.h.v.leeuwaarden@tue.nl

A.H. Löpker

e-mail: lopker@eurandom.tue.nl

T.J. Ott

Ott Associates, Chester, NJ, USA

e-mail: teun@teunisott.com 
transmission over the Internet. The process $X(t) \in \mathcal{M}$ has the following behavior. Between random collapse times $\left(\tau_{k}\right)_{k \in \mathbb{N}}$ the evolution is deterministic according to

$$
\frac{d}{d t} X(t)=r X(t)^{\alpha}, \quad r>0
$$

The random times $\tau_{k}$ are governed by a state-dependent Poisson process with rate $\lambda X(t)^{\beta}$ and $\beta>\alpha-1$, and at $\tau_{k}$ the process is multiplied by a random variable $Q_{k}$ in $[0,1)$ with distribution $F_{Q}(x)$, i.e., $X\left(\tau_{k}\right)=Q_{k} X\left(\tau_{k}-\right)$, where the random variables $\left(Q_{k}\right)_{k \in \mathbb{N}}$ are independent of $\left\{X(t): t \leq \tau_{k}\right\}$. If $\alpha=0$ and $\beta=0$, the PDMP $X(t)$ has a linear increase profile and independent losses and is known as the additive-increase multiplicative-decrease (AIMD) algorithm, also referred to as idealized TCP [21]. In general, if $X(0)=x$, the process $X(t)$ increases deterministically as

$$
\phi(x, t)= \begin{cases}\left(x^{1-\alpha}+(1-\alpha) r t\right)^{\frac{1}{1-\alpha}}, & \alpha \neq 1, \\ x e^{r t}, & \alpha=1,\end{cases}
$$

until the time $\tau_{1}$ of the first jump. It is clear that a larger $\alpha$ leads to a more aggressive increase profile. Also, a larger $\beta$ will lead to a more aggressive decrease profile, because jumps will occur more frequently. All possible combinations of $\alpha$ and $\beta$ together present a diverse pallet of increase-decrease profiles for the dynamics of TCP.

The dynamical behavior of TCP was originally modeled as a discrete-time Markov chain [21]. Several research papers $[11,15,17,18,20,21]$ deal with establishing scaling limits under a "low loss" scenario for such Markov Chains. Challenges were to establish weak convergence of processes to a limiting process, and to establish weak convergence of stationary distributions to the limiting stationary distribution.

Reference [17] studies a very general class of TCP control mechanisms for which it conjectures limiting behaviors. The limiting processes include what in this paper is $\alpha<1, \beta=0, Q=c$ with $c$ a constant. [18] proves weak convergence of stationary distributions for $\alpha<1, \beta=0, Q=c$ without establishing weak convergence of processes, but includes rate of convergence results. [20] proves all results conjectured in [17]: weak convergence of processes as well as of stationary distributions. [15] extends the results for $Q=c$ to a random $Q$. The class $\mathcal{M}$ is the class of scaling limits that occurs in [15].

In this paper we deal solely with the stationary behavior for processes in $\mathcal{M}$. Recent time-dependent results can be found in $[14,19]$. The key parameters in the description of $\mathcal{M}$ are $\alpha$ and $\beta$ for which we assume that $\beta>\alpha-1$. This is necessary to guarantee that the process is stable and admits a stationary distribution, cf. Theorem 1. Our contribution lies in the application of space-time transformations. We show that by a state-space transformation of the type $Y(t)=\gamma X(t)^{\delta}$ and a time transformation $Z(u)=X(t(u))$ with $t(u)=\int_{0}^{u} X(t(s))^{-v} d s$, stationary results for the process $X(t) \in \mathcal{M}\left(r, \alpha ; \lambda, \beta ; F_{Q}\right)$ follow directly from the stationary results for the idealized TCP process $Y(t) \in \mathcal{M}\left(1,0 ; \lambda, 0 ; F_{Q}\right)$. In this way, all known stationary results for idealized TCP can be transferred directly to results for any process in $\mathcal{M}$.

For a discussion of general PDMPs we refer to [7]. The processes in this paper belong to the special class of growth-collapse processes for which we refer to [6]. 
Growth-collapse processes are also referred to as stress release models [16, 22, 23]. There is also a close relationship to so-called repairable systems (see [13] and [12]). In [5] and [4] a different class of stress release models with additive jumps is discussed. The papers [24] and [12] give conditions for ergodicity of a very general class of growth collapse models, including our setting (model 4 in [24] and model 1 in [12]); the latter paper also gives conditions for non-explosiveness.

Space-time changes for general Markov processes can be found in the classical literature [9, 10]. For the special class of PDMPs in this paper, such space-time changes allow us to unify several earlier results. Ott et al. [21] use a space transformation to solve the idealized TCP case for "packet time" $(\alpha<1, \beta=0, Q=c)$ and use a time transformation to obtain the limiting stationary distribution for "clock time" $(\alpha=0, \beta=1, Q=c)$. Dumas et al. [8] consider the case $\alpha=0, \beta>-1$, and present the stationary distribution for $\beta=1$. Altman et al. [1] consider the case $\alpha<1$ and give an explicit analysis of $\beta=0,1$ using rather general mappings that involve both space and time transformations. Baccelli et al. [3] consider a more general class of models for non-persistent flows, that includes our model for the case $\alpha=0, \beta \leq 0$. They show that results for the throughput for $\beta>0$ follow from the case $\beta=0$ by applying appropriate substitutions to a differential equation. Maulik and Zwart [15] treat the case $\alpha \in[0,1]$ and $\beta>0$ and obtain explicit expressions for the stationary moments (cf. Sect. 3).

The remainder of this paper is structured as follows. In Sect. 2 we present our main results concerning the transformations that can be used to relate any two processes in $\mathcal{M}$. In Sect. 3 we combine a state-space transformation with a time transformation to express the stationary moments of $X(t)$ in terms of the stationary moments of idealized TCP. We also discuss the special cases of deterministic $Q$ and (generalized) uniform $Q$. As for the moments, the stationary distribution for idealized TCP then yields immediately the stationary distribution for any process $X(t) \in \mathcal{M}$. Some of the more formal proofs in this paper are provided in Appendices B and C, following a short introduction to the generator of the Markov process in Appendix A.

\section{Main results}

We denote by $q_{Q}(s)=\mathbb{E} Q^{s}=\mathbb{E} e^{-s \log (1 / Q)}$ the Laplace-Stieltjes transform of the non-negative random variable $\log (1 / Q)$. Hence, there exists an $s_{0} \leq 0$ such that $q_{Q}(s)$ is infinite for $s<s_{0}$ and finite for $s>s_{0}$. We also define the auxiliary function

$$
\psi_{Q}(s)=\frac{1-q_{Q}(s)}{s}, \quad s>0,
$$

and let $\psi_{Q}(0)=\lim _{s \rightarrow 0} \psi_{Q}(s)=\mathbb{E}(\log (1 / Q))$.

Next we properly define the class $\mathcal{M}$ of PDMPs.

Definition 1 Let $\mathcal{M}\left(r, \alpha ; \lambda, \beta ; F_{Q}\right)$ denote the class of PDMPs described in Sect. 1 . Here $r, \lambda>0$ are real constants, $F_{Q}$ is a distribution function on $[0,1]$, and $\alpha$ and $\beta$ are real constants with

$$
\beta>\alpha-1
$$


Moreover, $F_{Q}$ and $\alpha$ are chosen in a way such that $s_{0}<0$ and

$$
s_{0}<1-\alpha \text {. }
$$

The condition $s_{0}<0$ ensures that the distribution of $Q$ is reasonably well behaved in the sense that all moments $\mathbb{E}\left(\log (1 / Q)^{n}\right)$ are finite. Condition (3), which is redundant if $\alpha \leq 1$, is required to have a stable process.

We now first present some results for the stationary distribution that follow from the general theories of PDMPs and regenerative processes. Let $\Rightarrow$ denote convergence in distribution. For a random variable $A$ we denote throughout the paper by $\Pi_{A}(u)$ its distribution function, and by $\pi_{A}(u)$ its density.

Theorem 1 Let $X(t) \in \mathcal{M}\left(r, \alpha ; \lambda, \beta ; F_{Q}\right)$. Then $X(t)$ stays finite for finite $t$ and $X(t) \Rightarrow X$ as $t \rightarrow \infty$, where $X$ is a random variable with $X>0$ a.s. The distribution function $\Pi_{X}(u)$ of $X$ admits a density $\pi_{X}(u)$ satisfying

$$
\pi_{X}(u)=\frac{\lambda}{r} u^{-\alpha} \int_{u}^{\infty} y^{\beta} F_{Q}(u / y) \pi_{X}(y) d y .
$$

We give a proof of this theorem in Appendix B (see also [15]). The idea of the proof is to show that under conditions (2) and (3), the process has negative drift outside $[0, y]$ for some $y$ and positive drift inside $[0, w]$ for some $w \leq y$. It then follows that the regenerative process $X(t)$ has a finite cycle mean, which in turn proves the convergence result.

We next present a recursion relation for the moments of the random variable $X$ that follows the stationary distribution of $X(t)$.

Theorem 2 Let $X(t) \in \mathcal{M}\left(r, \alpha ; \lambda, \beta ; F_{Q}\right)$. If $q_{Q}(s-\alpha+1)<\infty$ for some $s \in \mathbb{R}$, then $\mathbb{E}\left(X^{s}\right)<\infty$ and

$$
\mathbb{E}\left(X^{s}\right)=\frac{\lambda}{r} \psi_{Q}(s-\alpha+1) \mathbb{E}\left(X^{s+\beta-\alpha+1}\right) .
$$

Theorem 2 is proved in Appendix C. Replacing the variable $s$ in (5) by $s+(k-$ 1) $(\beta-\alpha+1)$, with $k \geq 1$ an integer, gives upon iteration

$$
\mathbb{E}\left(X^{s+n(\beta-\alpha+1)}\right)=\mathbb{E}\left(X^{s}\right) \frac{r^{n}}{\lambda^{n}} \prod_{k=1}^{n} \frac{1}{\psi_{Q}(s+(k-1) \beta-k(\alpha-1))},
$$

which is particularly helpful for $s=0$.

We now introduce a state-space transformation $\mathcal{M} \rightarrow \mathcal{M}$ that preserves stationarity.

Proposition 1 (State-space transformation) Let $X(t) \in \mathcal{M}\left(r, \alpha ; \lambda, \beta ; F_{Q}\right)$ and define a new process $Y(t)=\gamma X(t)^{\delta}$ with $\delta>0$. Then

$$
Y(t) \in \mathcal{M}\left(r \delta \gamma^{\frac{1-\alpha}{\delta}}, 1+\frac{\alpha-1}{\delta} ; \lambda \gamma^{-\beta / \delta}, \frac{\beta}{\delta} ; F_{Q^{\delta}}\right) .
$$


Proof We have on the deterministic part of the trajectory

$$
\frac{d}{d t} Y(t)=r \gamma \delta X(t)^{\alpha+\delta-1}=r \delta \gamma^{\frac{1-\alpha}{\delta}} Y(t)^{1+\frac{\alpha-1}{\delta}}
$$

Let $\varphi(x)=\gamma x^{\delta}$, so that $Y(t)=\varphi(X(t))$. The probability of a jump during $[t, t+h]$ is given by

$$
\lambda \varphi^{-1}(Y(t))^{\beta} h+o(h)
$$

where $\varphi^{-1}$ denotes the inverse function of $\varphi$, and hence the jump intensity is given by $\lambda \gamma^{-\beta / \delta} Y(t)^{\beta / \delta}$. Further notice that

$$
\frac{Y\left(\tau_{k}-\right)}{Y\left(\tau_{k}\right)}=\frac{\gamma X\left(\tau_{k}-\right)^{\delta}}{\gamma X\left(\tau_{k}\right)^{\delta}}=Q^{\delta} .
$$

Condition (2) is invariant under the state-space transformation, since $\beta \geq \alpha-1$ iff $\beta / \delta \geq(\alpha-1) / \delta$. Letting $Q_{Y}:=Q^{\delta}$ and $q_{Q_{Y}}(s):=\mathbb{E}\left(Q_{Y}^{s}\right)=q_{Q}(\delta s)$, it is obvious that condition (3) is fulfilled for $Y(t)$.

The following result characterizes the relation between the stationary distributions of the processes $X(t)$ and $Y(t)$ that are both in $\mathcal{M}$.

Theorem 3 Let $X(t) \in \mathcal{M}\left(r, \alpha ; \lambda, \beta ; F_{Q}\right)$ and let $Y(t)=\gamma X(t)^{\delta}, \delta>0$ as in Proposition 1 . Let $X$ be the limit of $X(t)$ as given in Theorem 1. Then $Y(t) \Rightarrow Y \stackrel{d}{=} \gamma X^{\delta}$, where $Y$ has a distribution with density

$$
\pi_{Y}(x)=\pi_{X}\left((x / \gamma)^{1 / \delta}\right) \cdot \frac{x^{1 / \delta-1}}{\delta \gamma^{1 / \delta}}
$$

and $\mathbb{E}\left(Y^{s}\right)=\gamma^{s} \mathbb{E}\left(X^{s \delta}\right)$ for all s with $q_{Q}(s \delta-\alpha+1)=q_{Q_{Y}}\left(s-\left(\frac{\alpha-1}{\delta}+1\right)+1\right)<\infty$.

Proof The first part follows from the continuous mapping theorem, and the moment relation is trivial.

Next we define the random process

$$
\vartheta(t)=\int_{0}^{t} X(s)^{v} d s,
$$

for $t \geq 0$, and $v$ some real constant, and with inverse function

$$
\vartheta^{-1}(u)=\int_{0}^{u} \frac{1}{X\left(\vartheta^{-1}(s)\right)^{v}} d s .
$$

Throughout this section we use $u=\vartheta(t)$ and $t=\vartheta^{-1}(u)$. The time transformation of $X(t)$ is defined as follows. 
Proposition 2 (Time transformation) Let $v$ be a real number, such that

$$
q_{Q}(v-\alpha+1)<\infty .
$$

Suppose that $X(t) \in \mathcal{M}\left(r, \alpha ; \lambda, \beta ; F_{Q}\right)$. Define the time-changed process by $Z(u)=$ $X\left(\vartheta^{-1}(u)\right)$. Then

$$
Z(u) \in \mathcal{M}\left(r, \alpha-v ; \lambda, \beta-v ; F_{Q}\right) .
$$

Proof We have, in between two jumps,

$$
\frac{d}{d u} Z(u)=\frac{d}{d u} X\left(\vartheta^{-1}(u)\right)=r Z(u)^{\alpha-v} .
$$

Then

$\mathbb{P}(Z(u)$ jumps during $[u, u+h])=\mathbb{P}\left(X\left(\vartheta^{-1}(u)\right)\right.$ jumps during $\left.[u, u+h]\right)$.

Since $\vartheta^{-1}(u)=t$ and

$$
\vartheta^{-1}(u+h)=\vartheta^{-1}(u)+\int_{u}^{u+h} X\left(\vartheta^{-1}(s)\right)^{-v} d s=t+h Z(t)^{-v}+o(h),
$$

as $h \rightarrow 0$, it follows that

$$
\begin{aligned}
\mathbb{P}(Z(u) \text { jumps during }[u, u+h]) & =\mathbb{P}\left(X(t) \text { jumps during }\left[t, \vartheta^{-1}(u+h)\right]\right) \\
& =\lambda Z(t)^{\beta-v} h+o(h) .
\end{aligned}
$$

The intensity of the time-changed process is therefore given by $\lambda Z(t)^{\beta-v}$.

The jump sizes are not affected by a time change, hence leaving the jump distribution $F_{Q}$ unchanged, so condition (3) is still fulfilled for the time-changed process. Condition (2) remains unaltered under the time change, and (10) follows.

Note that $X(t)$ and $Z(t)$ are both regenerative processes, with cycles $C_{X}(y)$ and $C_{Z}(y)$ defined by successive visits to some fixed state $y$. We point out the following interesting property of the time-changed process, which links the cycle means to the stationary moments.

Proposition 3 Let $X(t) \in \mathcal{M}\left(r, \alpha ; \lambda, \beta ; F_{Q}\right)$ and let $Z(t)$ be the time-changed process as in Proposition 2, and assume that (9) holds. Then $\mathbb{E}\left(X^{v}\right)<\infty$, $\mathbb{E}\left(C_{X}(y)\right)<\infty, \mathbb{E}\left(C_{Z}(y)\right)<\infty$ and, for all $y>0$,

$$
\mathbb{E}\left(C_{Z}(y)\right)=\mathbb{E}\left(C_{X}(y)\right) \mathbb{E}\left(X^{v}\right) .
$$

Proof It is shown in Appendix B that under condition (2) the cycle $C_{X}(y)$ has a finite mean for some $y \in(0, \infty)$. We have $C_{Z}(y)=\vartheta\left(C_{X}(y)\right)=\int_{0}^{C_{X}(y)} d \vartheta(s)$ and hence

$$
\mathbb{E}\left(C_{Z}(y)\right)=\mathbb{E}\left(\vartheta\left(C_{X}(y)\right)\right)=\mathbb{E}\left(\int_{0}^{C_{X}(y)} X(s)^{v} d s\right)=\mathbb{E}\left(C_{X}(y)\right) \mathbb{E}\left(X^{v}\right),
$$


where the last equality follows from regeneration theory. Since $\mathbb{E}\left(X^{v}\right)<\infty$ by Theorem 2, we have that $\mathbb{E}\left(C_{Z}(y)\right)<\infty$.

Theorem 4 Let $X(t) \in \mathcal{M}\left(r, \alpha ; \lambda, \beta ; F_{Q}\right)$ and let $Z(\vartheta)=X(t)$ be the time-changed process as in Proposition 2. Let $X$ denote the limit of $X(t)$ given in Theorem 1. Then $Z(t) \Rightarrow Z$, and the distribution of $Z$ admits a density that satisfies

$$
\pi_{Z}(x)=\frac{x^{v}}{\mathbb{E}\left(X^{v}\right)} \pi_{X}(x)
$$

For all $s$ such that $q_{Q}(v+s-\alpha+1)<\infty$ the sth moment of $Z$ is given by

$$
\mathbb{E}\left(Z^{s}\right)=\frac{\mathbb{E}\left(X^{s+v}\right)}{\mathbb{E}\left(X^{v}\right)} .
$$

Proof In Appendix B it is shown that $\mathbb{E}\left(C_{X}(y)\right)<\infty$, so that by Proposition 3 we have that $\mathbb{E}\left(C_{Z}(y)\right)<\infty$. It follows that $Z(t)$ has a unique stationary distribution. Moreover, again by the well-known limit theorems for regenerative processes and Proposition 3,

$$
\begin{aligned}
\mathbb{P}(Z \leq y) & =\frac{\mathbb{E}\left(\int_{0}^{C_{Z}(y)} \mathbb{1}_{\{Z(s) \leq y\}} d s\right)}{\mathbb{E}\left(C_{Z}(y)\right)}=\frac{\mathbb{E}\left(\int_{0}^{\vartheta\left(C_{X}(y)\right)} \mathbb{1}_{\{Z(s) \leq y\}} d s\right)}{\mathbb{E}\left(C_{X}(y)\right) \mathbb{E}\left(X^{v}\right)} \\
& =\frac{\mathbb{E}\left(\int_{0}^{C_{X}(y)} \mathbb{1}_{\{X(t) \leq y\}} X(t)^{v} d t\right)}{\mathbb{E}\left(C_{X}(y)\right) \mathbb{E}\left(X^{v}\right)}=\frac{\mathbb{E}\left(\mathbb{1}_{\{X \leq y\}} X^{\nu}\right)}{\mathbb{E}\left(X^{v}\right)},
\end{aligned}
$$

so that (12) follows. If $q_{Q}(v+s-\alpha+1)<\infty$ then $\mathbb{E}\left(X^{s+v}\right)<\infty$ and formula (13) follows immediately from (12).

We conclude this section by a discussion on how to combine the introduced transformations. Denote by $V$ the admissible region in the $(\alpha, \beta)$-plane for which condition (2) is fulfilled. The transformations can be represented as mappings from $V \rightarrow V$, see Fig. 1, where the grey areas represent $V$. A state-space transformation is represented as a movement on radial lines, meeting in the point $(1,0)$, while a time change is represented as a movement parallel to the $\beta=\alpha-1$ line.

By using the transformations, each point $(\alpha, \beta)$ in $V$ can be mapped to any other point $\left(\alpha^{\prime}, \beta^{\prime}\right)$ in $V$ by first applying a state-space transformation as in Proposition 1 with

$$
\delta=\frac{\beta-\alpha+1}{\beta^{\prime}-\alpha^{\prime}+1}
$$

and then the time change in (10) with

$$
v=\beta \frac{\beta^{\prime}-\alpha^{\prime}+1}{\beta-\alpha+1}-\beta^{\prime} .
$$



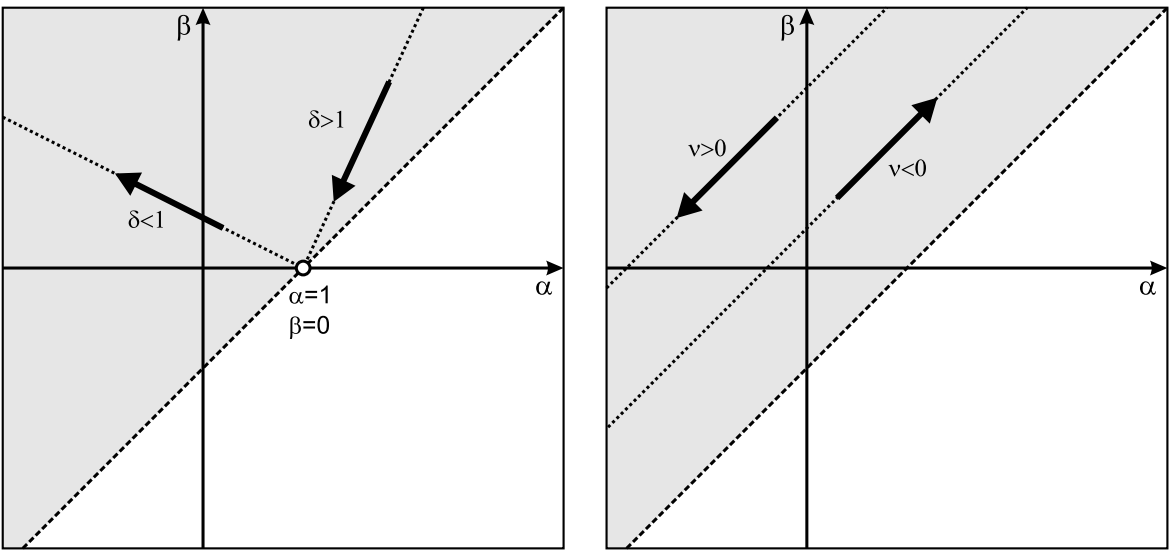

Fig. 1 State-space transformation (left) and time change (right) in the ( $\alpha, \beta)$-plane

Note that the order of the two transformations is important here. If first a time change is performed, the appropriate parameter is

$$
v=\beta-\frac{\beta-\alpha+1}{\beta^{\prime}-\alpha^{\prime}+1} \beta^{\prime},
$$

followed by the $\delta$ from (14) for the subsequent state-space transformation.

\section{Stationary moments and distribution}

With the transformations of Sect. 2 in our hands, the stationary results for one process in $\mathcal{M}\left(r, \alpha ; \lambda, \beta ; F_{Q}\right)$ yield stationary results for all other combinations of $\alpha, \beta$ in the admissible region $V$. In this section we shall use known results for the idealized TCP class $\mathcal{M}\left(1,0 ; \lambda, 0 ; F_{Q}\right)$ to derive expressions for the stationary moments and the stationary distribution of any process in the class $\mathcal{M}$.

Theorem 5 Let $X(t) \in \mathcal{M}\left(r, \alpha ; \lambda, \beta ; F_{Q}\right)$. Then

$$
\begin{aligned}
\mathbb{E}\left(X^{s}\right)= & \left(\frac{r(1-\alpha+\beta)}{\lambda}\right)^{\frac{s}{1-\alpha+\beta}} \frac{\Gamma\left(\frac{1-\alpha+s}{1-\alpha+\beta}\right)}{\Gamma\left(\frac{1-\alpha}{1-\alpha+\beta}\right)} \\
& \times \prod_{k=1}^{\infty} \frac{1-q_{Q}(s-\beta+k(1-\alpha+\beta))}{1-q_{Q}(-\beta+k(1-\alpha+\beta))},
\end{aligned}
$$

for all s for which $q_{Q}(s-\alpha+1)<\infty$.

Proof Let $Z(t) \in \mathcal{M}\left(1,0 ; \lambda, 0 ; F_{Q^{*}}\right)$ with $Q^{*}=Q^{1-\alpha+\beta}$. It is known that

$$
\mathbb{E}\left(Z^{s}\right)=\frac{\Gamma(s+1)}{\lambda^{s}} \prod_{k=1}^{\infty} \frac{1-q_{Q^{*}}(s+k)}{1-q_{Q^{*}}(k)},
$$


see $[11,14,21]$. Following Proposition 1 and (14), a state-space transformation with $\delta=1 /(1-\alpha+\beta)$ and $\gamma=(r(1-\alpha+\beta))^{1 /(1-\alpha+\beta)}$ maps $Z(t)$ to

$$
Y(t)=(r(1-\alpha+\beta) Z(t))^{1 /(1-\alpha+\beta)},
$$

with $Y(t) \in \mathcal{M}\left(r, \alpha-\beta ; \lambda, 0 ; F_{Q}\right)$. Since $q_{Q^{*}}(s)=q_{Q}(s(1-\alpha+\beta))$, we obtain

$$
\begin{aligned}
\mathbb{E}\left(Y^{s}\right)= & (r(1-\alpha+\beta))^{s /(1-\alpha+\beta)} \mathbb{E}\left(Z^{s /(1-\alpha+\beta)}\right) \\
= & \left(\frac{r(1-\alpha+\beta)}{\lambda}\right)^{s /(1-\alpha+\beta)} \Gamma\left(\frac{s}{1-\alpha+\beta}+1\right) \\
& \times \prod_{k=1}^{\infty} \frac{1-q_{Q}(s+k(1-\alpha+\beta))}{1-q_{Q}(k(1-\alpha+\beta))} .
\end{aligned}
$$

Applying a time transformation according to Proposition 2 and (15) with $v=-\beta$ then yields the process $X(t)$. Formula (16) follows immediately from (13).

Theorem 5 is due to Maulik and Zwart [15], who derive (16) by solving a difference equation for the Mellin transform. With $l=1-\alpha+\beta$ and $\gamma_{*}$ the Euler constant, they find that, for $s>\alpha-1, \alpha<1$ and $r=\lambda=1$,

$$
\begin{aligned}
\mathbb{E}\left(X^{s}\right)= & \left(l e^{-\gamma_{*}}\right)^{\frac{s}{l}} \frac{1-\alpha}{s+1-\alpha} \frac{1-q_{Q}(s+1-\alpha)}{1-q_{Q}(1-\alpha)} \\
& \times \prod_{k=1}^{\infty} e^{\frac{s}{k l}} \frac{1-q_{Q}(k l+s+1-\alpha)}{1-q_{Q}(k l+1-\alpha)} \frac{k l+1-\alpha}{k l+s+1-\alpha} .
\end{aligned}
$$

It can be easily verified that (16) and (19) correspond.

Let us next turn to the stationary distribution. In [11], Proposition 5, it is shown that for $Z(t) \in \mathcal{M}\left(1,0 ; \lambda, 0 ; F_{Q}\right)$ the Laplace-Stieltjes transform of the stationary distribution is given by

$$
\mathbb{E}\left(e^{-s Z}\right)=\mathbb{E}\left(\prod_{n=0}^{\infty} \frac{1}{1+(s / \lambda) \prod_{k=1}^{n} Q_{k}}\right),
$$

and alternatively (see [14])

$$
\mathbb{E}\left(e^{-s Z}\right)=\sum_{n=0}^{\infty} \frac{(-s / \lambda)^{n}}{\prod_{k=1}^{n}\left(1-q_{Q}(k)\right)}, \quad 0 \leq s \leq \lambda
$$

However, both expressions do not lead (in general) to tractable explicit representations for the stationary density. Fortunately, the classical case of deterministic jumps does give an explicit form for the density. 


\subsection{Deterministic jumps}

In the case where $Q$ is always a constant $c$, we have that $q_{Q}(u)=c^{s}$ and hence $s_{0}=-\infty$. Consequently all moments of $X$ exist, and from (4) we conclude that the stationary density fulfills

$$
\pi_{X}(u)=\frac{\lambda}{r u^{\alpha}} \int_{u}^{u / c} y^{\beta} \pi_{X}(y) d y .
$$

If $Z(t) \in \mathcal{M}\left(1,0 ; \lambda, 0 ; F_{Q^{*}}\right)$ then

$$
\pi_{Z}(u)=\lambda\left(\Pi_{Z}(u / c)-\Pi_{Z}(u)\right) .
$$

In [21] the solution to (22) was found to be

$$
\pi_{Z}(u)=\frac{\lambda}{(c ; c)_{\infty}} \sum_{k=0}^{\infty} \frac{(-1)^{k} c^{\frac{1}{2} k(k-1)}}{(c ; c)_{k}} \mathrm{e}^{-\lambda c^{-k} u},
$$

with $(q ; q)_{k}=\prod_{j=1}^{k}\left(1-q^{j}\right)$ the $q$-Pochhammer symbol. Alternatively, (23) can be written as

$$
\pi_{Z}(u)=\frac{\lambda}{(c ; c)_{\infty}} \sum_{k=0}^{\infty} \frac{c^{-k}}{\prod_{j=1}^{k}\left(c^{-j}-1\right)} \mathrm{e}^{-\lambda c^{-k} u},
$$

in which form the solution to (22) was derived in [8, 11].

We now employ our transformations to obtain the stationary distribution for the general $(\alpha, \beta)$-case.

Theorem 6 If $Q=c$ a.s. then

$$
\pi_{X}(x)=\frac{1}{r x^{\alpha}}\left(\frac{r(1-\alpha+\beta)}{\lambda}\right)^{\frac{\beta}{1-\alpha+\beta}} \frac{\pi_{Z}\left(\frac{x^{1-\alpha+\beta}}{r(1-\alpha+\beta)}\right)}{\Gamma\left(\frac{1-\alpha}{1-\alpha+\beta}\right)} \prod_{k=1}^{\infty} \frac{1-c^{k(1-\alpha+\beta)}}{1-c^{k(1-\alpha+\beta)-\beta}} .
$$

Proof Let $Z(t) \in \mathcal{M}\left(1,0 ; \lambda, 0 ; F_{Q^{*}}\right)$ with $Q^{*}=c^{1-\alpha+\beta}$. As in the proof of Theorem 5 we apply a state-space transformation with $\delta=1 /(1-\alpha+\beta)$ and $\gamma=$ $(r(1-\alpha+\beta))^{1 /(1-\alpha+\beta)}$ to $Z(t)$, yielding a process $Y(t) \in \mathcal{M}\left(r, \alpha-\beta ; \lambda, 0 ; F_{Q}\right)$, where $F_{Q}$ in this case is the distribution function having mass 1 at $c$. According to (7),

$$
\pi_{Y}(x)=\pi_{Z}\left(\frac{x^{1-\alpha+\beta}}{r(1-\alpha+\beta)}\right) \frac{x^{\beta-\alpha}}{r} .
$$

Next we perform a time change with $v=-\beta$, yielding a process $X(t)$ in the class $\mathcal{M}\left(r, \alpha ; \lambda, \beta ; F_{Q}\right)$. From (12) it follows that

$$
\pi_{X}(x)=\frac{x^{-\beta}}{\mathbb{E}\left(Y^{-\beta}\right)} \pi_{Y}(x) .
$$


According to (18), we have

$$
\mathbb{E}\left(Y^{-\beta}\right)=\left(\frac{\lambda}{r(1-\alpha+\beta)}\right)^{\frac{\beta}{1-\alpha+\beta}} \Gamma\left(\frac{1-\alpha}{1-\alpha+\beta}\right) \prod_{k=1}^{\infty} \frac{1-c^{k(1-\alpha+\beta)-\beta}}{1-c^{k(1-\alpha+\beta)}},
$$

and (25) follows.

\subsection{Another special case}

If $Q=U^{1 / \kappa}$, with some $\kappa>0$ and $U$ having a uniform distribution on [0,1], then

$$
q_{Q}(s)=\mathbb{E}\left(Q^{s}\right)=\int_{0}^{1} x^{s / \kappa} d x=\frac{\kappa}{\kappa+s},
$$

so that $s_{0}=-\kappa$ and $q_{Q}\left(s_{0}\right)=\infty$. It follows that $\mathbb{E}\left(X^{s}\right)<\infty$ if $s>\alpha-1-\kappa$.

Theorem 7 If $Q=U^{1 / \kappa}$ with $\kappa>0$ then

$$
\pi_{X}(x)=\frac{\Gamma\left(\frac{\kappa-\alpha+1}{\beta-\alpha+1}\right)}{\beta-\alpha+1}\left(\frac{\lambda}{r(\beta-\alpha+1)}\right)^{-\frac{\kappa-\alpha+1}{\beta-\alpha+1}} x^{\kappa-\alpha} \mathrm{e}^{-\frac{\lambda}{r(\beta-\alpha+1)} x^{\beta-\alpha+1}} .
$$

Proof We derive the proposition directly for all admissible $\alpha$ and $\beta$, without using the transformations. From (4) we obtain

$$
\pi_{X}(u)=\frac{\lambda u^{\kappa-\alpha}}{r} \int_{u}^{\infty} y^{\beta-\kappa} \pi_{X}(y) d y
$$

and hence

$$
\pi_{X}^{\prime}(u)=\left(\frac{\kappa-\alpha}{u}-\frac{\lambda u^{\beta-\alpha}}{r}\right) \pi_{X}(u) .
$$

For $y<x$,

$$
\pi_{X}(x)=\frac{\pi_{X}(y)}{y^{\kappa-\alpha}} x^{\kappa-\alpha} \exp \left(-\frac{\lambda}{r(\beta-\alpha+1)}\left(x^{\beta-\alpha+1}-y^{\beta-\alpha+1}\right)\right) .
$$

Letting $y \rightarrow 0$, and assuming that the limit $C=\lim _{y \rightarrow 0} \pi_{X}(y) y^{\alpha-\kappa}$ exists, we obtain

$$
\pi_{X}(x)=C x^{\kappa-\alpha} \exp \left(-\frac{\lambda}{r(\beta-\alpha+1)} x^{\beta-\alpha+1}\right) .
$$

The constant follows upon normalization.

We note that (27) was derived for the case $\kappa=\alpha=\beta=0$ in [11], p. 99.

Acknowledgement The work of JvL was supported by a VENI grant from The Netherlands Organization for Scientific Research (NWO). 
Open Access This article is distributed under the terms of the Creative Commons Attribution Noncommercial License which permits any noncommercial use, distribution, and reproduction in any medium, provided the original author(s) and source are credited.

\section{Appendix A: PDMPs, generator, domain}

Let $\mathbb{E}_{x}(\cdot)=\mathbb{E}(\cdot \mid X(0)=x)$. For bounded measurable functions $f$ the infinitesimal generator refers to the linear operator defined by the limit

$$
\mathcal{A}^{*} f(x)=\lim _{t \rightarrow 0}\left(\mathbb{E}_{x} f(X(t))-f(x)\right) / t,
$$

in the strong sense, that is with respect to the sup norm. It is well known that for functions for which this limit exists, the process

$$
f(X(t))-\int_{0}^{t} \mathcal{A}^{*} f(X(s)) d s
$$

is a martingale. The extended generator $\mathcal{A} f$ is a generalization of this operator and is defined as any measurable function $g$, for which $f(X(t))-\int_{0}^{t} g(X(s)) d s$ becomes a martingale. Following the exposition in [7] we see that our Markov process $X(t)$ is a PDMP and has the extended generator

$$
\mathcal{A} f(x)=r x^{\alpha} f^{\prime}(x)+\lambda x^{\beta} \int_{0}^{1}(f(x y)-f(x)) d F_{Q}(y) .
$$

For PDMPs Davis [7] has given criteria for a measurable function $f$ to belong to the domain $\mathcal{D}(\mathcal{A})$ of the extended generator. The domain $\mathcal{D}(\mathcal{A})$ contains all measurable functions $f: E \rightarrow \mathbb{R}$ for which $f$ is absolutely continuous and

$$
\mathbb{E}_{x} \sum_{i=1}^{N_{t}}\left|f\left(X\left(\tau_{i}-\right)\right)-f\left(X\left(\tau_{i}\right)\right)\right|<\infty, \quad \forall x \in[0, \infty), t \geq 0 .
$$

If $f$ is absolutely continuous and locally bounded on $[0, \infty)$ then $f \in \mathcal{D}(\mathcal{A})$ follows immediately from the fact that according to (1) $X(t)$ has a deterministic upper bound. Since the domain of $\mathcal{A}$ is probably much larger, it is desirable to know whether $\mathcal{D}(\mathcal{A})$ comprises function that are not locally bounded. From Lemma 1 in [14] we obtain the following result:

Lemma 1 (Domain) The function $f_{s}(x)=x^{s}$ is in the domain $\mathcal{D}(\mathcal{A})$ if $q_{Q}(s)<\infty$.

We define the counting process $N_{t}=\inf \left\{n \in \mathbb{N}: \tau_{n} \geq t\right\}$. In order to use the methodology of PDMPs we have to assure that $\mathbb{E}\left(N_{t}\right)<\infty$.

Lemma $2 \mathbb{E}_{x} N_{t}<\infty$ for all $t>0, x \geq 0$. 
Proof If $\beta=0$ then the jump intensity is just $\lambda$ and clearly $\mathbb{E} N_{t}=\lambda t$ is finite for all $t>0$. The general case with $\beta>0$ can be transformed via a time change as described in Propositions 2 and 3 to the $\beta=0$ case (see Fig. 1).

Next we show that the process will not escape to infinity in finite time.

Lemma $3 T_{\infty}:=\sup \{t>0: X(t)<\infty\}=\infty$ a.s.

Proof If $\alpha<1$ then this follows from the fact that $\phi(x, t)$ is finite for all $t>0$ and $X(t) \leq \phi(x, t)$. If $\alpha \geq 1$, a time change with $v=\beta$ transforms the process into a process $Z(t)$ with increase rate parameter $\alpha-\beta<1$ and constant jump intensity (see Proposition 2). $T_{\infty}=\infty$ iff

$$
\vartheta^{-1}(u)=\int_{0}^{u} \frac{1}{Z(s)^{\beta}} d s<\infty
$$

for all $u>0$. Since $N_{t}<\infty$ for all $t$ (see Lemma 2), we have $\min \{Z(s): 0 \leq s \leq$ $t\}>0$ and hence $\vartheta^{-1}(u) \leq u(\min \{Z(s): 0 \leq s \leq t\})^{-\beta}<\infty$.

\section{Appendix B: Proof of Theorem 1}

Lemma 3 proves that $X(t)$ stays finite for finite $t$. We now prove Theorem 1 for the case $\beta=0$. The general form of Theorem 1 then follows by applying a time change as described in Propositions 2 and 3.

Theorem 8 Let $X(t) \in \mathcal{M}\left(r, \alpha ; \lambda, 0 ; F_{Q}\right)$. Then $X(t) \Rightarrow X$ as $t \rightarrow \infty$, where $X$ is a random variable with $X>0$ a.s. The distribution function $\Pi_{X}(u)$ of $X$ admits a density $\pi_{X}(u)$ satisfying

$$
\pi_{X}(u)=\frac{\lambda}{r} u^{-\alpha} \int_{u}^{\infty} F_{Q}(u / y) \pi_{X}(y) d y .
$$

We need two lemmas and the following definitions for the proof of Theorem 8 . Let $T_{y}^{*}=\inf \{t>0: X(t) \leq y\}$ and $T_{y}=\inf \{t>0: X(t)=y\}$ denote the first time the process jumps below $y$ and the first hitting time of $y$. Note that we have $\alpha<1$ throughout the proof.

Lemma 4 There is a $y \in(0, \infty)$ such that $\mathbb{E}_{x} T_{y}^{*}<\infty$ for all $x \geq y$.

Proof Recall that $f_{s}(x)=x^{s}$ and that $f_{s} \in \mathcal{D}(\mathcal{A})$ if $q_{Q}(s)<\infty$. We first show that there is an $s>0$, such that $\limsup _{x \rightarrow \infty} \mathcal{A} f_{s}(x)<0$ as $x \rightarrow \infty$. We have

$$
\mathcal{A} f_{s}(x)=s r x^{\alpha+s-1}-\lambda x^{s}\left(1-q_{Q}(s)\right)=x^{s}\left(s r x^{\alpha-1}-\lambda\left(1-q_{Q}(s)\right)\right) .
$$

Let $s>0$, then $x^{s} \rightarrow \infty$ and $x^{\alpha-1} \rightarrow 0$ as $x \rightarrow \infty$, hence $\mathcal{A} f_{s}(x) \rightarrow-\infty$ and thus $\limsup _{x \rightarrow \infty} \mathcal{A} f_{s}(x)<0$ (note that $\left(1-q_{Q}(s)\right)>0$ since $s>0$ ). 
We have shown that there is a $y \in(0, \infty)$, such that $\mathcal{A} f_{s}(x)<-\varepsilon$ for all $x \geq y$ and some $s>0$. Pick one such $x$ and recall from the definition of the extended generator that the process $M_{t}=f_{s}(X(t))-f_{s}(x)-\int_{0}^{t} \mathcal{A} f_{s}(X(s)) d s$ is a zero-mean martingale. Optional sampling yields $\mathbb{E}_{x} M_{t \wedge T_{y}^{*}}=0$, and hence

$$
0=\mathbb{E}_{x}\left(f_{s}\left(X\left(t \wedge T_{y}^{*}\right)\right)-f_{s}(x)-\int_{0}^{t \wedge T_{y}^{*}} \mathcal{A} f_{s}(X(s)) d s\right) .
$$

It follows that $\mathbb{E}_{x}\left(f_{s}\left(X\left(t \wedge T_{y}^{*}\right)\right)-f_{s}(x)+\left(t \wedge T_{y}^{*}\right) \varepsilon\right) \leq 0$. Consequently,

$$
\mathbb{E}_{x}\left(t \wedge T_{y}^{*}\right) \leq \frac{1}{\varepsilon}\left(f_{s}(x)-\mathbb{E}_{x} f_{s}\left(X\left(t \wedge T_{y}^{*}\right)\right)\right) \leq \frac{f_{s}(x)}{\varepsilon}
$$

Letting $t \rightarrow \infty$ it follows that $\mathbb{E}_{x} T_{y}^{*}<\infty$.

Next we show that the mean of the hitting time $\mathbb{E}_{x} T_{w}$ is also finite for some $w$.

Lemma 5 There is a $w \in(0, \infty)$ such that $\mathbb{E}_{x} T_{w}<\infty$ for all $x \leq y$.

Proof It is enough to show that Lemma 4 holds for the reciprocal process $R(t)=$ $X^{-1}(t)$, which is the case if we can show that $\limsup _{x \rightarrow \infty} \mathcal{A}_{R} f_{s}(x)<0$, where

$$
\mathcal{A}_{R} f(x)=-r x^{2-\alpha} f^{\prime}(x)+\lambda \int_{0}^{1}(f(x / y)-f(x)) d F_{Q}(y)
$$

is the generator of the Markov process $R(t)$. By following the proof of Lemma 1 it is clear that $f_{s}$ is in the domain of $\mathcal{A}_{R}$ if $q_{Q}(-s)<\infty$. We have

$$
\begin{aligned}
\mathcal{A}_{R} f_{S}(x) & =-s r x^{1-\alpha+s}+\lambda x^{s}\left(q_{Q}(-s)-1\right) \\
& =x^{1-\alpha+s}\left(-s r+\lambda x^{\alpha-1}\left(q_{Q}(-s)-1\right)\right) .
\end{aligned}
$$

Let $0<s<-s_{0}$. Then $f_{s}$ is in the domain of $\mathcal{A}_{R}$. Since $x^{1-\alpha+s} \rightarrow \infty$ and $x^{\alpha-1} \rightarrow 0$ as $x \rightarrow \infty$, it follows that $\limsup _{x \rightarrow \infty} \mathcal{A}_{R} f_{s}(x)<0$. The result then follows along the lines of the second part of the proof of Lemma 4.

Proof of Theorem 8 The process $X(t)$ is a regenerative process with regeneration cycles $C_{X}(y)$ starting at upcrossings of $y$.

Under the stated conditions the assertions of Lemmas 4 and 5 are both fulfilled, i.e. there are $y, w \in(0, \infty)$ such that $\mathbb{E}_{x} T_{y}^{*}<\infty$ for all $x \geq y$ and $\mathbb{E}_{x} T_{w}<\infty$ for all $x \leq y$. Without loss of generality we can assume that $w<y$.

We will show that $\mathbb{E}_{w} T_{y}<\infty$. Then $\mathbb{E}_{x} T_{y}$ for all $x \leq y$ and we have already shown that under the given conditions $\mathbb{E}_{y} T_{y}^{*}<\infty$. It follows then that for the regenerative process $X(t)$, with regeneration cycles starting at upcrossings of $y$, the cycle length has a finite mean and the assertion of the lemma follows.

To prove $\mathbb{E}_{w} T_{y}<\infty$ consider a process $W(t)$ which behaves similarly to $X(t)$ if $X(t) \in[0, \infty) \backslash[w, y]$, but has the following behavior if $W(t) \in[w, y]$ : it jumps with intensity $\lambda$ and if there is a jump at time $t$ then $W(t)=w \wedge(W(t-) \cdot Q)$. We 
can construct $X(t)$ and $W(t)$ on a common probability space, such that $X(t) \geq W(t)$ w.p. one. Consequently, $W(t)$ will reach $y$ later than $X(t)$, so it remains to show that, starting in $W(0)=w$, the expected time to reach $y$ is finite.

Starting in $w$ the process $W(t)$ will reach $y$ without any jump with some positive probability, or it jumps down into the set $[0, w]$. We proved in Lemma 4 that the time until the process returns to $w$ has finite mean. It follows that the time until $W(t)$ reaches $y$ is a geometric sum of random variables with finite mean, and hence has finite mean.

We have seen that the regeneration cycles $C_{X}(y)$ of $X(t)$ have finite mean, hence $X(t) \Rightarrow X$ as $t \rightarrow \infty$ follows from regeneration theory (see e.g. [2]). But we have actually shown more. Under the given conditions, Lemma 5 shows that also the reciprocal process $R(t)=X^{-1}(t)$ tends to a finite limit, hence $X$ is not zero.

To prove (4) we use the fact that $\int_{0}^{\infty} \mathcal{A} f(x) \pi_{X}(x) d x=0$ for bounded functions $f \in \mathcal{D}(\mathcal{A})$. Let $g_{n}(x)=n \int_{0}^{x} \mathbb{1}_{\{w \in[u-1 / n, u+1 / n]\}} d w$, then $g_{n}$ is bounded and absolutely continuous. We have

$$
\int_{0}^{\infty} r x^{\alpha} g_{n}^{\prime}(x) \pi_{X}(x) d x=n \int_{u-1 / n}^{u+1 / n} r x^{\alpha} \pi_{X}(x) d x
$$

which tends to $r u^{\alpha} \pi_{X}(u)$ as $n \rightarrow \infty$. Since $g_{n}(x) \rightarrow \mathbb{1}_{\{x \geq z\}}$, we obtain

$$
\begin{aligned}
\int_{0}^{\infty} & \left(\lambda \int_{0}^{1}\left(g_{n}(x y)-g_{n}(x)\right) d F_{Q}(y)\right) \pi_{X}(x) d x \\
& \rightarrow \int_{0}^{\infty} \lambda\left(\int_{0}^{1} \mathbb{1}_{\{x y \geq u\}} d F_{Q}(y)-\mathbb{1}_{\{x \geq u\}}\right) \pi_{X}(x) d x \\
& =\int_{u}^{\infty} \lambda\left(\int_{u / x}^{1} d F_{Q}(y)-1\right) \pi_{X}(x) d x=-\int_{u}^{\infty} \lambda F_{Q}(u / x) \pi_{X}(x) d x .
\end{aligned}
$$

Hence (31) follows.

Having proved Theorem 8 the final step to establish Theorem 1 is an application of the time change, which transforms the $\beta=0$ case to the $\beta \neq 0$ case (Proposition 2 with $v=\beta$ ). Note that according to Proposition 3, we have to fulfill (9), which yields the extra condition (3) in Definition 1.

\section{Appendix C: Proof of Theorem 2}

We shall now prove that $\mathbb{E}_{x}\left(X^{s}\right)<\infty$ if $q_{Q}(s-\alpha+1)<\infty$ and that (5) holds. Let $f_{s, w}(x)=\mathbb{1}_{\{x \leq w\}} x^{s-\alpha+1}$. Since $q_{Q}(s-\alpha+1)<\infty$, it follows from Lemma 1 that $f_{s, w} \in \mathcal{D}(\mathcal{A})$. Then

$$
\begin{aligned}
\mathcal{A} f_{s, w}(x)= & \left(r(s-\alpha+1) x^{s}+\lambda\left(q_{Q}(s-\alpha+1)-1\right) x^{s+\beta-(\alpha-1)}\right) \mathbb{1}_{\{x \leq w\}} \\
& +\left(\lambda x^{s+\beta-(\alpha-1)} \int_{0}^{w / x} y^{s-\alpha+1} d F_{Q}(y)\right) \mathbb{1}_{\{x>w\}} .
\end{aligned}
$$


Since $f_{s, w}$ is bounded, it follows from $\int_{0}^{\infty} \mathcal{A} f(x) \pi_{X}(x) d x=0$ that

$$
\int_{0}^{w} x^{s} \pi_{X}(x) d x=\frac{\lambda}{r} \psi_{Q}(s-\alpha+1) \int_{0}^{w} x^{s+\beta-(\alpha-1)} \pi_{X}(x) d x-C(w),
$$

where $C(w)=\frac{\lambda}{r(s-\alpha+1)} \int_{w}^{\infty} x^{s+\beta-(\alpha-1)} \int_{0}^{w / x} y^{s-\alpha+1} d F_{Q}(y) \pi_{X}(x) d x$. We have

$$
\int_{0}^{w / x} y^{s-\alpha+1} d F_{Q}(y) \leq \int_{0}^{1} y^{s-\alpha+1} d F_{Q}(y)=q_{Q}(s-\alpha+1),
$$

and hence

$$
0 \leq C(w) \leq \frac{\lambda q_{Q}(s-\alpha+1)}{r(s-\alpha+1)} \int_{w}^{\infty} x^{s+\beta-(\alpha-1)} \pi_{X}(x) d x .
$$

We see that $C(w) \rightarrow 0$ when $w \rightarrow \infty$. Consequently, relation (5) follows from (33) by dominated convergence.

To prove that $\mathbb{E}_{x}\left(X^{s}\right)<\infty$, we first assume that $s=0$. Then $q_{Q}(s-\alpha+1)=$ $q_{Q}(1-\alpha)<\infty$ and since $\mathbb{E}_{X}\left(X^{s}\right)=1<\infty$ it follows that $\mathbb{E}_{x}\left(X^{\beta-(\alpha-1)}\right)<\infty$. We thus have $\mathbb{E}_{x}\left(X^{t}\right)<\infty$ for all $t \in[0, \beta-(\alpha-1)]$ and by induction $\mathbb{E}_{x}\left(X^{t}\right)<\infty$ for all $t>0$.

On the other hand, if $s<0$ and $q_{Q}(s-\alpha+1)<\infty$, then there is a $k \in \mathbb{N}$, such that $s_{k}=s+k(\beta-(\alpha-1)) \geq 0$ and hence $\mathbb{E}_{x}\left(X^{s_{k}}\right)<\infty$. Moreover, $s_{k}-\beta \geq s-\alpha+1$ and also $q_{Q}\left(s_{k}-\beta\right)<\infty$. It follows then from $\mathbb{E}_{x}\left(X^{s_{k}}\right)<\infty$ that $\mathbb{E}_{x}\left(X^{s_{k-1}}\right)<\infty$ and by induction $\mathbb{E}_{x}\left(X^{s}\right)<\infty$.

\section{References}

1. Altman, A., Avrachenkov, K., Kherani, A.A., Prabhu, B.J.: Performance analysis and stochastic stability of congestion control protocols. In: Proceedings of IEEE Infocom 2005 (2005)

2. Asmussen, S.: Applied Probability and Queues, 2nd edn. Springer, New York (2003)

3. Baccelli, F., Kim, K.B., McDonald, D.: Equilibria of a class of transport equations arising in congestion control. Queueing Syst. 55, 1-8 (2007)

4. Borovkov, K., Vere-Jones, D.: Explicit formulae for stationary distributions of stress release processes. J. Appl. Probab. 37, 315-321 (2000)

5. Borovkov, K., Novikov, A.: On a piece-wise deterministic Markov process model. Stat. Probab. Lett. 53, 421-428 (2001)

6. Boxma, O., Perry, D., Stadje, W., Zacks, S.: A Markovian growth-collapse model. Adv. Appl. Probab. 38, 221-243 (2006)

7. Davis, M.H.A.: Markov Models and Optimization. Chapman \& Hall, London (1993)

8. Dumas, V., Guillemin, F., Robert, Ph.: A Markovian analysis of Additive-Increase MultiplicativeDecrease (AIMD) algorithms. Adv. Appl. Probab. 34, 85-111 (2002)

9. Dynkin, E.B.: Markov Processes I. Springer, New York (1965)

10. Ethier, S.N., Kurtz, T.G.: Markov Processes. Characterization and Convergence. Wiley, New York (1986)

11. Guillemin, F., Robert, Ph., Zwart, B.: AIMD algorithms and exponential functionals. Ann. Appl. Probab. 14, 90-117 (2004)

12. Last, G.: Ergodicity properties of stress release, repairable system and workload models. Adv. Appl. Probab. 36, 471-498 (2004)

13. Last, G., Szekli, R.: Time and Palm stationarity of repairable systems. Stoch. Process. Their Appl. 79, $17-43(1999)$ 
14. Löpker, A.H., van Leeuwaarden, J.S.H.: Transient moments of the TCP window size process. J. Appl. Probab. 45, 163-175 (2008)

15. Maulik, K., Zwart, B.: An extension of the square root law of TCP. Ann. Oper. Res. 170(1), 217-232 (2009)

16. Ogata, J., Vere-Jones, D.: Inference for earthquake models: a self-correcting model. Stoch. Process. Their Appl. 17, 337-347 (1984)

17. Ott, T.J.: Transport protocols in the TCP paradigm and their performance. Telecommun. Syst. 30, 351-385 (2005)

18. Ott, T.J.: Rate of convergence for the square root formula in the Internet transmission control protocol. Adv. Appl. Probab. 38, 1132-1154 (2006)

19. Ott, T.J., Kemperman, J.H.B.: The transient behavior of processes in the TCP paradigm. Probab. Eng. Inf. Sci. 22, 431-471 (2008)

20. Ott, T.J., Swanson, J.: Asymptotic behavior of a generalized TCP congestion avoidance algorithm. J. Appl. Probab. 44, 618-635 (2007)

21. Ott, T.J., Kemperman, J.H.B., Mathis, M.: The stationary behavior of ideal TCP congestion avoidance. Unpublished manuscript (1996). Available at http://www.teunisott.com/Papers/TCP_Paradigm

22. Vere-Jones, D.: Earthquake prediction: a statistician's view. J. Phys. Earth 26, 129-146 (1978)

23. Vere-Jones, D.: On the variance properties of stress release models. Aust. J. Stat. 30, 123-135 (1988)

24. Zheng, X.: Ergodic theorems for stress release processes. Stoch. Process. Their Appl. 37, 239-258 (1991) 